\title{
Análisis De Los Fundamentos Básicos Técnicos Del Baloncesto En EI Rendimiento Deportivo De Los Estudiantes Categoría Intermedia Del Distrito Chambo - Riobamba
}

\author{
Karolina Janeth Cando Brito \\ Magíster en Cultura Física y Entrenamiento Deportivo \\ Docente en Unidad Educativa Riobamba, Ecuador \\ Henry Rodolfo Gutierrez Cayo \\ Magister en Entrenamiento Deportivo \\ Docente Universidad Nacional de Chimborazo, Ecuador \\ Hernán Leopoldo Ponce Bravo PhD \\ Actividad Física y Salud \\ Docente Universidad Nacional de Chimborazo, Ecuador \\ Helder Guillermo Aldas Arcos \\ Magister en Entrenamiento Deportivo \\ Catedrático de la Universidad Católica de Cuenca, Ecuador \\ Orlando David Mazón Moreno \\ Magister en Cultura Física y Entrenamiento Deportivo \\ Docente de la Escuela Superior Politécnica de Chimborazo, Ecuador
}

doi: 10.19044/esj.2017.v13n26p60 URL:http://dx.doi.org/10.19044/esj.2017.v13n26p60

\begin{abstract}
This research was carried out on student's athletes of Circuito Educativo 06D01C02 Riobamba-Chambo, in Chimborazo province district. The main objective of this study is to know the basic technical fundamentals of basketball in the performance of students of the intermediate category. This however would be used to determine specifically the basics technical basis. It would also help to define the level of the basic technical fundamentals in order to make a proposal. The elaboration of a training plan is aimed at developing the technical basis for the intermediate category. Within the methodology, it is a quantitative and qualitative research. This is a field research because it will run in the scene where practical assessments will be applied to each one of the students. Additionally, it will be a documentary bibliographical mode. It will be exploratory and correlational, and would take into account the population and sample which comprises of 6 coaches, 10 monitors, and 75 students of the category. The sample was
\end{abstract}


under direct observation technique. As an instrument, the survey and a closed questionnaire was applied. Later on, the processing and tabulation alternative hypothesis was accepted. It was expressed as conclusions that the basic technical foundations in this category are determined by the work of dribbling, shooting ring tray, chest pass, and defense as predominant elements. It appears that the level of the basic technical fundamentals on student is regular, which is due to inadequate training and poor methods in the teaching process.

Keywords: Basketball, development, sports, teaching, skills, technical basis

\section{Resumen}

La investigación se realizó con los estudiantes deportistas del circuito educativo 06D01C02 del distrito Chambo - Riobamba provincia de Chimborazo tuvo como objetivo principal, conocer los fundamentos básicos técnicos del baloncesto en el rendimiento de los estudiantes deportistas categoría intermedia y de esta manera específicamente, determinar los fundamentos básicos técnicos, definir el nivel de los fundamentos técnicos básicos para realizar como propuesta, la elaboración de una planificación del entrenamiento orientada al desarrollo de los fundamentos técnicos de la categoría intermedia, dentro de la metodología es una investigación cuantitativa y cualitativa es una investigación de campo por qué se ejecutó en el lugar de los hechos, donde se tomó las evaluaciones prácticas de cada uno de los estudiantes del mismo modo bibliográfico documental, de tipo exploratorio y correlacional, tomando la muestra, a quienes se aplicó la técnica de la observación directa, como técnica la encuesta y un cuestionario cerrado, posteriormente al procesamiento y tabulación de datos se aceptó la hipótesis alternativa, expresándose como conclusiones que los fundamentos básicos técnicos en esta categoría se ven determinados por el trabajo del Dribling, Tiro al aro en bandeja, pase de pecho y la defensa como elementos predominantes, y el deficiente proceso metodológico al momento de su enseñanza.

Palabras-claves: Baloncesto, desarrollo deportivo, didáctica, habilidades, fundamento

\section{Introducción}

El tema a desarrollar se origina en la necesidad de mejorar los fundamentos básicos técnicos del baloncesto y su incidencia que tiene en el desarrollo deportivo de los estudiantes deportistas, tanto en la técnica individual, como en lo físico, además que el buen dominio de los fundamentos es muy necesario en la edad deportiva, con un buen manejo de 
estos elementos podemos evitar muchas errores mediante situaciones de juego y lo más importante tener una vida diaria plena y placentera, y un buen estado físico.

Ecuador (2010), a nivel nacional la practica del deporte es uno de los objetivos en los cuales se enfoca el desarrollo integral de los estudiantes, mediante las técnicas de enseña docente - alumno se forman programas en los cuales se formen las actividades tanto físicas como intelectuales, prueba de ello es la enseñanza de los fundamentos básicos técnicos de baloncesto que se contextualiza como el periodo en que un principiante, por lo general un niño, se inicia en el proceso de formación deportivo a largo plazo.

En Ecuador anualmente son miles los niños que se inician en el Baloncesto, sin embargo pocos llegan al alto nivel competitivo. En ocasiones se observa que los estudiantes son sometidos a programas de entrenamiento típicos de adultos, sin considerarse que en edades intermedias debe primar el aprendizaje de la técnica y con ella el dominio de las habilidades motrices típicas del deporte (Ecuador, A. N, 2010).

Diversos autores del plano internacional y nacional opinan que los fundamentos básicos técnicos debe estar muy matizada por los juegos y el aprendizaje de ejercicios diversos, muy asociados con la técnica deportiva y que el estudiante adquiere un nivel óptimo de preparación física para su edad, como resultado de la influencia de los ejercicios técnicos que aprende, debiendo primar la preparación.

El presente estudio brinda una alternativa de solución para la problemática, planteando el análisis orientado al desarrollo de los fundamentos técnicos en la categoría intermedia para los estudiantes del circuito 06D01C02 distrito Chambo - Riobamba, de la provincia de Chimborazo, y que puede ser adecuado para mejorar el nivel de rendimiento en el baloncesto a la vez que se formule una propuesta metodológica que podría ser utilizada para el mejoramiento de los fundamentos básicos técnicos de baloncesto. El presente trabajo de Investigación se desarrolló y aplicó durante el período Noviembre 2014 - Junio 2015.

\section{Objetivos}

- Analizar los fundamentos básicos técnicos de los estudiantes deportistas de la categoría intermedia del circuito 06D01C02 distrito Chambo - Riobamba, de la provincia de Chimborazo.

- Verificar el nivel del rendimiento deportivo del baloncesto en los estudiantes deportistas de la categoría intermedia del circuito 06D01C02 distrito Chambo - Riobamba, de la provincia de Chimborazo.

\section{Metodología}

La presente investigación es de carácter cuantitativo y cualitativo. 
Es Cuantitativa por cuanto se utilizan procesos matemáticos (valores o datos estadísticos), para estos datos que serán aplicadas las encuestas que arrojarán datos de la investigación.

Es Cualitativa porque se va a valorar los fundamentos básicos técnicos de baloncesto y su influencia en el rendimiento deportivo de los estudiantes categoría intermedia del circuito 06D01C02 distrito ChamboRiobamba de la Provincia de Chimborazo.

El trabajo de investigación se ejecutó en el lugar de los hechos, donde se tomó las evaluaciones prácticas de cada uno de los estudiantes de categoría intermedia del circuito.

Tabla 1. Plan de Recolección de Información

\begin{tabular}{|c|c|}
\hline PREGUNTAS BÁSICAS & EXPLICACIÓN \\
\hline ¿Para qué? & Para alcanzar los objetivos de investigación. \\
\hline ¿De qué personas u objetos? & $\begin{array}{c}\text { Profesores de CC.FF } \\
\text { Entrenadores } \\
\text { Deportistas }\end{array}$ \\
\hline ¿Sobre qué aspecto? & $\begin{array}{c}\text { Los fundamentos básicos técnicos del baloncesto y su } \\
\text { rendimiento deportivo }\end{array}$ \\
\hline ¿Quién? ¿Quiénes? & Grupo de investigación. \\
\hline ¿A quiénes? & Entrenadores \\
& Estudiantes-Deportistas \\
\hline ¿Cuándo? & Período Escolar 2015 \\
\hline ¿Dónde? & Circuito 06D01C02 Distrito Chambo-Riobamba de la \\
& Provincia de Chimborazo \\
\hline ¿Cuántas veces? & Dos veces ( Piloto y la de validación) \\
\hline ¿Qué técnicas de recolección? & Medición y valoración cuantitativa \\
\hline ¿Con que? & Encuesta \\
\hline
\end{tabular}

Elaborado por: Grupo de investigación

Para esta investigación se utilizara la encuesta porque nos permite visualizar los todos los aspectos relacionados con la investigación realizada (Anexo 1).

\section{Población y Muestra}

Tomados en consideración: entrenadores, y estudiantes categoría intermedia del circuito 06D01C02 distrito Chambo-Riobamba de la Provincia de Chimborazo son en total 510 el universo de estudio:

\section{Fórmula}

$$
\begin{aligned}
& \mathrm{n}=\frac{\mathrm{N}}{(\mathbf{e})^{2}(\mathrm{~N}-1)+1} \\
& \mathrm{n}=\frac{510}{(0.07)^{2}(510-1)+1} \\
& \mathrm{n}=\frac{510}{(0.0049)(509)+1}
\end{aligned}
$$




$$
\begin{aligned}
\mathrm{n} & =\frac{510}{2.4941+1} \\
\mathrm{n} & =\frac{510}{3.4941} \\
\mathbf{n} & =\mathbf{1 4 6}
\end{aligned}
$$

Tabla 2. Muestra

\begin{tabular}{|c|c|c|}
\hline Población & Muestra & Porcentaje \\
\hline Estudiantes deportistas & 140 & $96 \%$ \\
\hline Entrenadores & 6 & $4 \%$ \\
\hline Total & 146 & $100 \%$ \\
\hline
\end{tabular}

Nota: Número de encuestas aplicadas a cada segmento de estudio

\section{Desarrollo de la Investigación}

Análisis de datos e interpretación de resultados

Resultados de la encuesta aplicada a los entrenadores

Pregunta 1. ¿La exactitud de los fundamentos técnicos de baloncesto permite el mejor desenvolvimiento en el juego?

Tabla 3.

\begin{tabular}{|c|c|c|c|}
\hline Cód. & Alternativas & Frecuencia & $\%$ \\
\hline 1 & Siempre & 4 & 66,67 \\
\hline 2 & Casi siempre & 0 & 0,00 \\
\hline 3 & Nunca & 2 & 33,33 \\
\hline \multicolumn{2}{|c|}{ Total } & 6 & 100 \\
\hline
\end{tabular}

Nota: Datos de la encuesta aplicada a los entrenadores del circuito 06D01C02

\section{Análisis de Datos}

En la presente se alcanza determinar que el $67 \%$ de los encuestados manifiestan que siempre la exactitud fundamentos técnicos del baloncesto permite el mejor desenvolvimiento del juego, mientras que el otro 33\% manifiesta que casi siempre.

\section{Interpretación de Resultados}

Esto significa que las dos terceras partes de consideran que la exactitud de los fundamentos básicos técnicos del baloncesto, permiten el mejor desenvolvimiento en el juego, por lo que se deberá trabajar en el desarrollo y perfeccionamiento de los fundamentos, debido a que las acciones de juego se desarrollan en fracciones de segundos.

Pregunta 2. ¿Usted como entrenador considera que la rapidez de movimiento permite ejecutar las acciones con eficiencia y eficacia? 
Tabla 4.

\begin{tabular}{|c|c|c|c|}
\hline Cód. & Alternativas & Frecuencia & $\%$ \\
\hline 1 & Siempre & 5 & 83,33 \\
\hline 2 & Casi siempre & 0 & 0,00 \\
\hline 3 & Nunca & 1 & 16,67 \\
\hline \multicolumn{2}{|c|}{ Total } & 6 & 100 \\
\hline
\end{tabular}

Nota: Datos de la encuesta aplicada a los entrenadores del circuito 06D01C02

\section{Análisis de Datos}

En la presente figura claramente se puede determinar que el $83 \%$ de los encuestados consideran que la rapidez de movimiento permite ejecutar las acciones de manera eficiente y eficaz, mientras que el otro $17 \%$ considera que nunca.

\section{Interpretación de Resultados}

Esto significa que las tres cuartas partes de entrenadores valoran la importancia de ejecutar los movimientos técnicos con rapidez, y una cuarta parte que no lo considera, mismas acciones que en un momento considerable del juego permitirán al deportista ejecutar con eficiencia y eficacia, para resolver situaciones en cuestión de segundos.

Pregunta 3. ¿Cree usted como entrenador que el dribling, el tiro, y los pases son indispensables en el juego?

Tabla 5.

\begin{tabular}{|c|c|c|c|}
\hline Cód. & Alternativas & Frecuencia & $\%$ \\
\hline 1 & Siempre & 5 & 83,33 \\
\hline 2 & Casi siempre & 0 & 0,00 \\
\hline 3 & Nunca & 1 & 16,67 \\
\hline \multicolumn{2}{|c|}{ Total } & 6 & 100 \\
\hline
\end{tabular}

Nota: Datos de la encuesta aplicada a los entrenadores del circuito 06D01C02

\section{Análisis de Datos}

En la presente figura claramente se puede determinar que el $83 \%$ de los encuestados consideran que siempre, el entrenador debe impartir los conocimientos del dribling, el tiro y los pases, y el 17\% mencionan que casi siempre y consideran otros factores como más importantes.

\section{Interpretación de Resultados}

Esto significa que tres cuartas partes de los entrenadores que siempre consideran indispensables los elementos técnicos como el dribling, el tiro y los pases, dentro del juego, para mejorar su desempeño en el juego, mientras que una cuarta parte que no lo considera indispensable.

Pregunta 4. ¿El desarrollo de los fundamentos técnicos del baloncesto permite el buen desempeño en el campo de juego? 
Tabla 6.

\begin{tabular}{|c|c|c|c|}
\hline Cód. & Alternativas & Frecuencia & $\%$ \\
\hline 1 & Siempre & 4 & 66,67 \\
\hline 2 & Casi siempre & 0 & 0,00 \\
\hline 3 & Nunca & 2 & 33,33 \\
\hline \multicolumn{2}{|c|}{ Total } & 6 & 100 \\
\hline
\end{tabular}

Nota: Datos de la encuesta aplicada a los entrenadores del circuito 06D01C02

\section{Análisis de Datos}

En la presente figura claramente se puede determinar que el $67 \%$ de los encuestados consideran que siempre, el entrenador debe desarrollar los fundamentos técnicos para el correcto desempeño al momento del juego y el $33 \%$ mencionan que casi siempre sería importante el desarrollo de los fundamentos técnicos.

\section{Interpretación de Resultados}

Esto significa que un poco más de la mitad de entrenadores, consideran importante el desarrollo de los fundamentos técnicos estimulan la práctica de los mismos en los deportistas dentro y fuera de los entrenamientos, mientras que menos de la mitad consideran que nunca, permiten el buen desempeño en el campo de juego.

Pregunta 5. ¿Cree usted que el deportista debe conocer acerca del arbitraje? Tabla 7.

\begin{tabular}{|c|c|c|c|}
\hline Cód. & Alternativas & Frecuencia & $\%$ \\
\hline 1 & Siempre & 5 & 83,33 \\
\hline 2 & Casi siempre & 0 & 0,00 \\
\hline 3 & Nunca & 1 & 16,67 \\
\hline \multicolumn{2}{|c|}{ Total } & 6 & 100 \\
\hline
\end{tabular}

Nota: Datos de la encuesta aplicada a los entrenadores del circuito 06D01C02

\section{Análisis de Datos}

En la presente figura claramente se puede determinar que el $83 \%$ de los encuestados consideran que siempre, el Docente considera que el deportista debe conocer sobre las reglas del arbitraje mientras que el 17\% mencionan que casi siempre el deportista debe conocer el reglamento.

\section{Interpretación de Resultados}

Esto significa que en su mayoría los entrenadores consideran de vital importancia la enseñanza del reglamento de baloncesto, fundamentalmente para que los deportistas no puedan cometer errores que vayan en contra del equipo mientras que un solo entrenador manifiesta que no es muy importante la enseñanza de la reglamentación y que eso sería un proceso que el deportista aprende durante el desarrollo del juego. 
Pregunta 6. ¿Cómo indicador potencial la técnica influye en el rendimiento deportivo?

Tabla 8

\begin{tabular}{|c|c|c|c|}
\hline Cód. & Alternativas & Frecuencia & $\%$ \\
\hline 1 & Siempre & 5 & 83,33 \\
\hline 2 & Casi siempre & 0 & 0,00 \\
\hline 3 & Nunca & 1 & 16,67 \\
\hline \multicolumn{2}{|c|}{ Total } & 6 & 100 \\
\hline
\end{tabular}

Nota: Datos de la encuesta aplicada a los entrenadores del circuito 06D01C02

\section{Análisis de Datos}

En la presente figura claramente se puede determinar que el $83 \%$ de los encuestados consideran que siempre, el indicador potencial influye en el rendimiento, y el $17 \%$ mencionan que casi siempre.

\section{Interpretación de Resultados}

Esto significa que las tres cuartas partes de los entrenadores están de acuerdo que la técnica es significativa en el rendimiento deportivo, debido a que en actualidad el alto grado de dominio técnico es evidencia de un mejor rendimiento deportivo personal y para el equipo. Mientras que una cuarta parte de los entrenadores mencionan que nunca la técnica influye en el rendimiento.

Pregunta 7. ¿Cree usted que la fuerza, velocidad y resistencia son capacidades física predominantes del rendimiento deportivo?

Tabla 9.

\begin{tabular}{|c|c|c|c|}
\hline Cód. & Alternativas & Frecuencia & $\%$ \\
\hline 1 & Siempre & 4 & 66,67 \\
\hline 2 & Casi siempre & 0 & 0,00 \\
\hline 3 & Nunca & 2 & 33,33 \\
\hline \multicolumn{2}{|c|}{ Total } & 6 & 100 \\
\hline
\end{tabular}

Nota: Datos de la encuesta aplicada a los entrenadores del circuito 06D01C02

\section{Análisis de Datos}

En la presente figura claramente se puede determinar que el $67 \%$ de los encuestados consideran que siempre, que los entrenadores desarrollan las capacidades físicas predominantes, y el 33\% mencionan que nunca.

\section{Interpretación de Resultados}

Esto significa que las dos terceras partes de entrenadores creen que las capacidades físicas predominantes influyen en el rendimiento deportivo, ya que el desarrollo de la fuerza, velocidad y resistencia son predominantes ayudan a mejorar su condición física, mientras una mínima parte que nunca influyen las capacidades físicas en el rendimiento deportivo. 
Pregunta 8. ¿Considera importante trabajar la flexibilidad para mejorar el rendimiento deportivo en los estudiantes?

Tabla 10.

\begin{tabular}{|c|c|c|c|}
\hline Cód. & Alternativas & Frecuencia & $\%$ \\
\hline 1 & Siempre & 4 & 66,67 \\
\hline 2 & Casi siempre & 0 & 0,00 \\
\hline 3 & Nunca & 2 & 33,33 \\
\hline \multicolumn{2}{|c|}{ Total } & 6 & 100 \\
\hline
\end{tabular}

Nota: Datos de la encuesta aplicada a los entrenadores del circuito 06D01C02

\section{Análisis de Datos}

En la presente figura claramente se puede determinar que el $67 \%$ de los encuestados consideran que siempre, el trabajo de la flexibilidad es importante para mejorar el rendimiento deportivo, mientras que el 33\% considera menos importe el desarrollo de la flexibilidad.

\section{Interpretación de Resultados}

Lo que significa que más de la mitad de entrenadores conocen el beneficio de la flexibilidad al momento del juego, pese a no ser una capacidad determínate durante el juego, produce beneficios en la enseñanza del gesto técnico y en la competencia, mientras que menos de la mitad de entrenadores consideran que la flexibilidad no es importante trabajar.

Pregunta 9. ¿La preparación psicológica permite una buena estabilización en el rendimiento deportivo?

Tabla 11.

\begin{tabular}{|c|c|c|c|}
\hline Cód. & Alternativas & Frecuencia & $\%$ \\
\hline 1 & Siempre & 3 & 50,00 \\
\hline 2 & Casi siempre & 0 & 0,00 \\
\hline 3 & Nunca & 3 & 50,00 \\
\hline \multicolumn{2}{|c|}{ Total } & 6 & 100 \\
\hline
\end{tabular}

Nota: Datos de la encuesta aplicada a los entrenadores del circuito 06D01C02

\section{Análisis de Datos}

En la presente figura claramente se puede determinar que el $50 \%$ de los encuestados consideran que siempre, la preparación psicológica permite una buena estabilización en el rendimiento deportivo mientras que el otro $50 \%$ manifiesta que casi siempre es importante el uso de la psicología para la estabilización.

\section{Interpretación de Resultados}

Esto significa que la mitad de entrenadores conocen los beneficios del trabajo psicológico en el proceso de formación deportiva orientada hacia el alto rendimiento y la importancia de una estabilización psicológica antes, 
durante y después de la preparación competitiva, al contrario la mitad de los entrenadores manifiestan que nunca la preparación psicológica permite una buena estabilidad en el rendimiento deportivo.

Pregunta 10. ¿Cree usted que la aplicación de un plan de entrenamiento mejora su rendimiento deportivo?

Tabla 12.

\begin{tabular}{|c|c|c|c|}
\hline Cód. & Alternativas & Frecuencia & $\%$ \\
\hline 1 & Siempre & 5 & 83,33 \\
\hline 2 & Casi siempre & 0 & 0,00 \\
\hline 3 & Nunca & 6 & 16,67 \\
\hline \multicolumn{2}{|c|}{ Total } & 6 & 100 \\
\hline
\end{tabular}

Nota: Datos de la encuesta aplicada a los entrenadores del circuito 06D01C02

\section{Análisis de Datos}

En la presente figura claramente se puede determinar que el $83 \%$ de los encuestados consideran que siempre, se debe aplicar un plan de entrenamiento orientado al desarrollo de los fundamentos básicos técnicos, mientras que el $17 \%$ menciona que casi siempre.

\section{Interpretación de Resultados}

Esto significa que más de la mitad de entrenadores consideran necesario utilizar una herramienta metodológica y saben los beneficios de un plan de entrenamiento orientado al desarrollo de los fundamentos básicos técnicos los mismos que seleccionados y dosificados de una manera adecuada mejorara el rendimiento deportivo de los deportistas.

\section{Análisis de datos e interpretación de resultados}

\section{Resultados de la encuesta aplicada a los estudiantes}

Pregunta 1. ¿La exactitud de los fundamentos técnicos de baloncesto permite el mejor desenvolvimiento en el juego?

Tabla 13.

\begin{tabular}{|c|c|c|c|}
\hline Cód. & Alternativas & Frecuencia & $\%$ \\
\hline 1 & Siempre & 125 & 89,29 \\
\hline 2 & Casi siempre & 1 & 0,71 \\
\hline 3 & Nunca & 14 & 10,00 \\
\hline \multicolumn{2}{|c|}{ Total } & 140 & 100 \\
\hline
\end{tabular}

Nota: Datos de la encuesta aplicada a los estudiantes del circuito 06D01C02

\section{Análisis de Datos}

Los resultados del grafico nos indica que el $89 \%$ de los encuestados consideran que siempre, la exactitud de los fundamentos técnicos del baloncesto permite mejorar el desenvolvimiento durante el juego, un $1 \%$ expresa que casi siempre, mientras que el 10\%manifiesta que nunca es 
importante la exactitud de los fundamentos técnicos durante el desenvolvimiento del juego.

\section{Interpretación de Resultados}

Esto significa más de la mitad de estudiantes valoran la calidad de realizar con exactitud los fundamentos técnicos y su importancia en el desenvolvimiento durante el juego lo que posiblemente les permitirá un mejor rendimiento durante el juego. Mientras que menos de la mitad que nunca la aplicación de un plan de entrenamiento influye en su rendimiento deportivo.

Pregunta $\mathbf{N}^{\circ}$ 2. ¿Usted como deportista considera que la rapidez de movimiento permite ejecutar las acciones con eficiencia y eficacia?

Tabla 14.

\begin{tabular}{|c|c|c|c|}
\hline Cód. & Alternativas & Frecuencia & $\%$ \\
\hline 1 & Siempre & 138 & 98,57 \\
\hline 2 & Casi siempre & 1 & 0,71 \\
\hline 3 & Nunca & 1 & 0,71 \\
\hline \multicolumn{2}{|c|}{ Total } & 140 & 100 \\
\hline
\end{tabular}

Nota: Datos de la encuesta aplicada a los estudiantes del circuito 06D01C02

\section{Análisis de Datos}

En la presente figura claramente se puede determinar que el $98 \%$ de los encuestados consideran que siempre la rapidez permite ejecutar las acciones con eficiencia y eficacia mientras el $1 \%$ expresa que casi siempre y el otro $1 \%$ manifiesta que nunca.

\section{Interpretación de Resultados}

Esto significa que las tres cuartas partes los deportistas consideran que la rapidez de movimiento les permitirá ejecutar las acciones con eficiencia y eficacia la misma que dentro del campo de juego se pondrá de manifiesto en un momento clave y decisivo, mientras que la mínima parte considera que la rapidez de movimiento permite ejecutar acciones con eficiencia y eficacia.

Pregunta 3. ¿Cree usted como deportista que el dribling, el tiro, y los pases son indispensables en el juego?

Tabla 15.

\begin{tabular}{|c|c|c|c|}
\hline Cód. & Alternativas & Frecuencia & $\%$ \\
\hline 1 & Siempre & 137 & 97,86 \\
\hline 2 & Casi siempre & 1 & 0,71 \\
\hline 3 & Nunca & 2 & 1,43 \\
\hline \multicolumn{2}{|c|}{ Total } & 140 & 100 \\
\hline
\end{tabular}

Nota: Datos de la encuesta aplicada a los estudiantes del circuito 06D01C02 


\section{Análisis de Datos}

En la presente figura claramente se puede determinar que el $98 \%$ de los encuestados consideran que siempre, los fundamentos con el dribling, el tiro y los pases son indispensables durante el juego mientras que el $1 \%$ manifiesta que casi siempre y el otro $1 \%$ que nunca son indispensables durante el juego.

\section{Interpretación de Resultados}

Esto significa que los deportistas consideran importante el Dribling, el tiro y los pases como fundamentos técnicos indispensables al momento del juego, mientras que una pequeña parte casi siempre y nunca lo que es visible que en su mayoría están de acuerdo trabajar estos fundamentos.

Pregunta 4. ¿El desarrollo de los fundamentos técnicos del baloncesto permite el buen desempeño en el campo de juego?

Tabla 16.

\begin{tabular}{|c|c|c|c|}
\hline Cód. & Alternativas & Frecuencia & $\%$ \\
\hline 1 & Siempre & 138 & 98,57 \\
\hline 2 & Casi siempre & 1 & 0,71 \\
\hline 3 & Nunca & 1 & 0,71 \\
\hline \multicolumn{2}{|c|}{ Total } & 140 & 100 \\
\hline
\end{tabular}

Nota: Datos de la encuesta aplicada a los estudiantes del circuito 06D01C02

\section{Análisis de Datos}

En la presente figura claramente se puede determinar que el $98 \%$ de los encuestados consideran que siempre, el desarrollo de los fundamentos técnicos del baloncesto permite el buen desempeño en el campo de juego un $1 \%$ casi siempre y el otro $1 \%$ nunca.

\section{Interpretación de Resultados}

Esto significa que la mayoría de los deportistas consideran la importancia del desarrollo de los fundamentos técnicos lo cual les permitirá desempeñarse de mejor manera en el campo de juego y marcar una diferencia en el dominio de los mismos durante las competencias.

Pregunta 5. ¿Cree usted que el deportista debe conocer acerca del arbitraje?

\begin{tabular}{|c|c|c|c|}
\hline \multicolumn{4}{|c|}{ Tabla 17.} \\
\hline Cód. & Alternativas & Frecuencia & $\%$ \\
\hline 1 & Siempre & 133 & 95,00 \\
\hline 2 & Casi siempre & 1 & 0,71 \\
\hline 3 & Nunca & 6 & 4,29 \\
\hline \multicolumn{2}{|c|}{ Total } & 140 & 100 \\
\hline
\end{tabular}

Nota: Datos de la encuesta aplicada a los estudiantes del circuito 06D01C02 


\section{Análisis de Datos}

En la presente figura claramente se puede determinar que el $95 \%$ de los encuestados consideran que siempre, el deportista debe conocer acerca del arbitraje el $1 \%$ casi siempre y el otro $1 \%$ nunca.

\section{Interpretación de Resultados}

Esto significa que la gran parte de deportistas consideran de suma importancia el conocimiento de las reglas del arbitraje para el correcto desenvolvimiento en el campo de juego y no cometer errores técnicos al momento de la competencia y lo principal no salir del campo de juego por la acumulación de faltas técnicas.

Pregunta 6. ¿Cómo indicador potencial la técnica influye en el rendimiento deportivo?

Tabla 18.

\begin{tabular}{|c|c|c|c|}
\hline Cód. & Alternativas & Frecuencia & $\%$ \\
\hline 1 & Siempre & 134 & 95,71 \\
\hline 2 & Casi siempre & 2 & 1,43 \\
\hline 3 & Nunca & 4 & 2,86 \\
\hline \multicolumn{2}{|c|}{ Total } & 140 & 100 \\
\hline
\end{tabular}

Nota: Datos de la encuesta aplicada a los estudiantes del circuito 06D01C02

\section{Análisis de Datos}

En la presente figura claramente se puede determinar que el $96 \%$ de los encuestados consideran que siempre, la técnica influye en el rendimiento deportivo como un indicador potencial un $1 \%$ casi siempre y un $3 \%$ nunca.

\section{Interpretación de Resultados}

Esto significa que la mayoría de los deportistas y consideran el concepto de mejorar la técnica como un indicador potencial que influye en el rendimiento deportivo dicho de otro modo la técnica va a contribuir a la economía del gasto energético y por ende un mejor rendimiento en el campo de juego.

Pregunta 7. ¿Cree usted que la fuerza, velocidad y resistencia son capacidades física predominantes del rendimiento deportivo?

Tabla 19.

\begin{tabular}{|c|c|c|c|}
\hline Cód. & Alternativas & Frecuencia & $\%$ \\
\hline 1 & Siempre & 134 & 95,71 \\
\hline 2 & Casi siempre & 2 & 1,43 \\
\hline 3 & Nunca & 4 & 2,86 \\
\hline \multicolumn{2}{|c|}{ Total } & 140 & 100 \\
\hline
\end{tabular}

Nota: Datos de la encuesta aplicada a los estudiantes del circuito 06D01C02 


\section{Análisis de Datos}

En la presente figura claramente se puede determinar que el $96 \%$ de los encuestados consideran que siempre, un $1 \%$ casi siempre y un $3 \%$ que nunca.

\section{Interpretación de Resultados}

Esto significa que la mayoría de los deportistas consideran importe el entrenamiento de las capacidades físicas como la fuerza, velocidad y resistencia como capacidades predominantes y la base para el futuro desarrollo de capacidades físicas que beneficiaran su rendimiento deportivo.

Pregunta 8. ¿Considera importante trabajar la flexibilidad para mejorar el rendimiento deportivo en los estudiantes?

Tabla 20.

\begin{tabular}{|c|c|c|c|}
\hline Cód. & Alternativas & Frecuencia & $\%$ \\
\hline 1 & Siempre & 133 & 95,00 \\
\hline 2 & Casi siempre & 1 & 0,71 \\
\hline 3 & Nunca & 6 & 4,29 \\
\hline \multicolumn{2}{|c|}{ Total } & 140 & 100 \\
\hline
\end{tabular}

Nota: Datos de la encuesta aplicada a los estudiantes del circuito 06D01C02

\section{Análisis de Datos}

En la presente figura claramente se puede determinar que el $95 \%$ de los encuestados consideran que siempre, el trabajo de la flexibilidad para mejorar el rendimiento deportivo en los estudiantes un $1 \%$ casi siempre y un $4 \%$ nunca.

\section{Interpretación de Resultados}

Esto significa que la mayoría de los deportistas consideran importante el trabajo de la flexibilidad para mejorar el rendimiento deportivo misma que no se manifestara en el campo de juego, pero servirá para el mejoramiento de las ejecuciones técnicas en las competencias, como en los entrenamientos.

Pregunta 9. ¿La preparación psicológica permite una buena estabilización en el rendimiento deportivo?

Tabla 21.

\begin{tabular}{|c|c|c|c|}
\hline Cód. & Alternativas & Frecuencia & $\%$ \\
\hline 1 & Siempre & 134 & 95,71 \\
\hline 2 & Casi siempre & 3 & 2,14 \\
\hline 3 & Nunca & 3 & 2,14 \\
\hline \multicolumn{2}{|c|}{ Total } & 140 & 100 \\
\hline
\end{tabular}

Nota: Datos de la encuesta aplicada a los estudiantes del circuito 06D01C02 


\section{Análisis de Datos}

En la presente figura claramente se puede determinar que el $96 \%$ considera que siempre la psicología es importante en la estabilización psicológica, un $2 \%$ considera que casi siempre y un $2 \%$ nunca.

\section{Interpretación de Resultados}

Esto significa que la mayoría de los deportistas consideran que el trabajo psicológico ayuda a mejorar sus resultados competitivos y controlar el pre arranque dentro de la competencia.

Pregunta 10. ¿Cree usted que la aplicación de un plan de entrenamiento mejora su rendimiento deportivo?

\begin{tabular}{|c|c|c|c|}
\hline \multicolumn{5}{|c|}{ Tabla 22.} \\
\hline Cód. & Alternativas & Frecuencia & $\%$ \\
\hline 1 & Siempre & 115 & 82,14 \\
\hline 2 & Casi siempre & 7 & 5,00 \\
\hline 3 & Nunca & 18 & 12,86 \\
\hline \multicolumn{2}{|c|}{ Total } & 140 & 100 \\
\hline
\end{tabular}

Nota: Datos de la encuesta aplicada a los estudiantes del circuito 06D01C02

\section{Análisis de Datos}

En la presente figura claramente se puede determinar que el $82 \%$ siempre ha conocido sobre un plan de entrenamiento orientado al desarrollo de los fundamentos técnicos del baloncesto un 5\% casi siempre y un $13 \%$ nunca.

\section{Interpretación de Resultados}

Esto significa lo importante de aplicar un plan de entrenamiento orientado al desarrollo de los fundamentos básicos técnicos del baloncesto y creen importante una correcta planificación para elevar el rendimiento deportivo de los deportistas.

\section{Verificación de la Hipótesis}

Selección del nivel de significación: Para la verificación de la hipótesis se utilizara el nivel de significación del $\infty=0.05$

Verificación: La verificación de los datos aportados ha sido positiva, se comprueba que los fundamentos técnicos básicos del baloncesto inciden en el rendimiento deportivo. 


\section{Prueba del Chi-Cuadrado}

\section{Planteamiento de la Hipótesis}

\section{Modelo Lógico}

$H_{0}$. Los fundamentos básicos técnicos del baloncesto no inciden en el rendimiento deportivo de los estudiantes categoría intermedia del circuito 06d01c02 distrito Chambo-Riobamba, provincia de Chimborazo.

$H_{1}$. Los fundamentos básicos técnicos del baloncesto si inciden en el rendimiento deportivo de los estudiantes categoría intermedia del circuito 06d01c02 distrito Chambo-Riobamba, provincia de Chimborazo.

Para la realización del Chi-cuadrado se tomó en cuenta el total de las preguntas, 10 de la variable independiente, y 10 de la variable dependiente para la comprobación respectiva.

\section{Modelo Matemático}

$$
\begin{aligned}
& H_{0}=\mathrm{O}=\mathrm{E} \\
& H_{1}=\mathrm{O} \neq \mathrm{E}
\end{aligned}
$$

\section{Modelo Estadístico}

Fórmula:

$$
X^{2}=\sum\left[\frac{(O-E)^{2}}{E}\right]
$$

$\mathrm{X}^{2}=$ Ji cuadrado
$\sum=$ Sumatoria.
$\mathrm{O}=$ Frecuencias Observadas.
$\mathrm{E}=$ Frecuencias Esperadas

\section{Nivel de Significación}

Se utilizará el nivel $\infty=0,05$ de confianza.

Para decidir sobre estas regiones primeramente determinamos los grados de libertad conociendo que el cuadro está formado por 2 filas y 3 columnas.

$$
\begin{aligned}
& \mathrm{gl}=(\mathrm{f}-1) \cdot(\mathrm{c}-1) \\
& \mathrm{gl}=(2-1) \cdot(3-1) \\
& \mathrm{gl}=1 \times 2=2
\end{aligned}
$$

$$
X_{t}^{2}=5.99
$$

Entonces con $2 \mathrm{gl} \mathrm{y}$ un nivel de 0,05 tenemos en la tabla el valor de 5,99. Tabla 23.

\begin{tabular}{|c|c|c|c|c|c|}
\hline & \multicolumn{5}{|c|}{ Probabilidad de un valor superior - Alfa $(\alpha)$} \\
\hline Grados libertad & 0,1 & 0,05 & 0,025 & 0,01 & 0,005 \\
\hline 1 & 2,71 & 3,84 & 5,02 & 6,63 & 7,88 \\
\hline 2 & 4,61 & $\underline{5,99}$ & 7,38 & 9,21 & 10,6 \\
\hline
\end{tabular}

Nota: Datos sobre los grados de libertad aplicados 


\section{Descripción de la Población}

Para el cálculo de Ji-cuadrado, se toma la población establecida anteriormente en el capítulo tercero, en relación al problema de investigación, así tenemos:

Tabla 24.

\begin{tabular}{|c|c|c|}
\hline Población & Muestra & Porcentaje \\
\hline Entrenadores del circuito 06D01C02 & 6 & $4 \%$ \\
\hline Estudiantes deportistas del circuito 06D01C02 & 140 & $96 \%$ \\
\hline
\end{tabular}

Nota: Porcentaje y número de encuestas aplicadas al estudio

\section{Zona de Aceptación y Rechazo}

Entonces con $\mathbf{2}$ gl y un nivel de $\mathbf{0 , 0 5}$ tenemos en la tabla de $\mathbf{X}^{\mathbf{2}}$ el valor de 5,99: Por consiguiente se acepta la hipótesis nula para todo valor de ji cuadrado que se encuentre hasta el valor 5,99 y se rechaza la hipótesis nula cuando los valores calculados son mayores a 5,99 y se acepta la hipótesis alternativa.

Se acepta $H_{1}$ si: $\quad X_{t}^{2} \geq \mathbf{5 . 9 9}$.

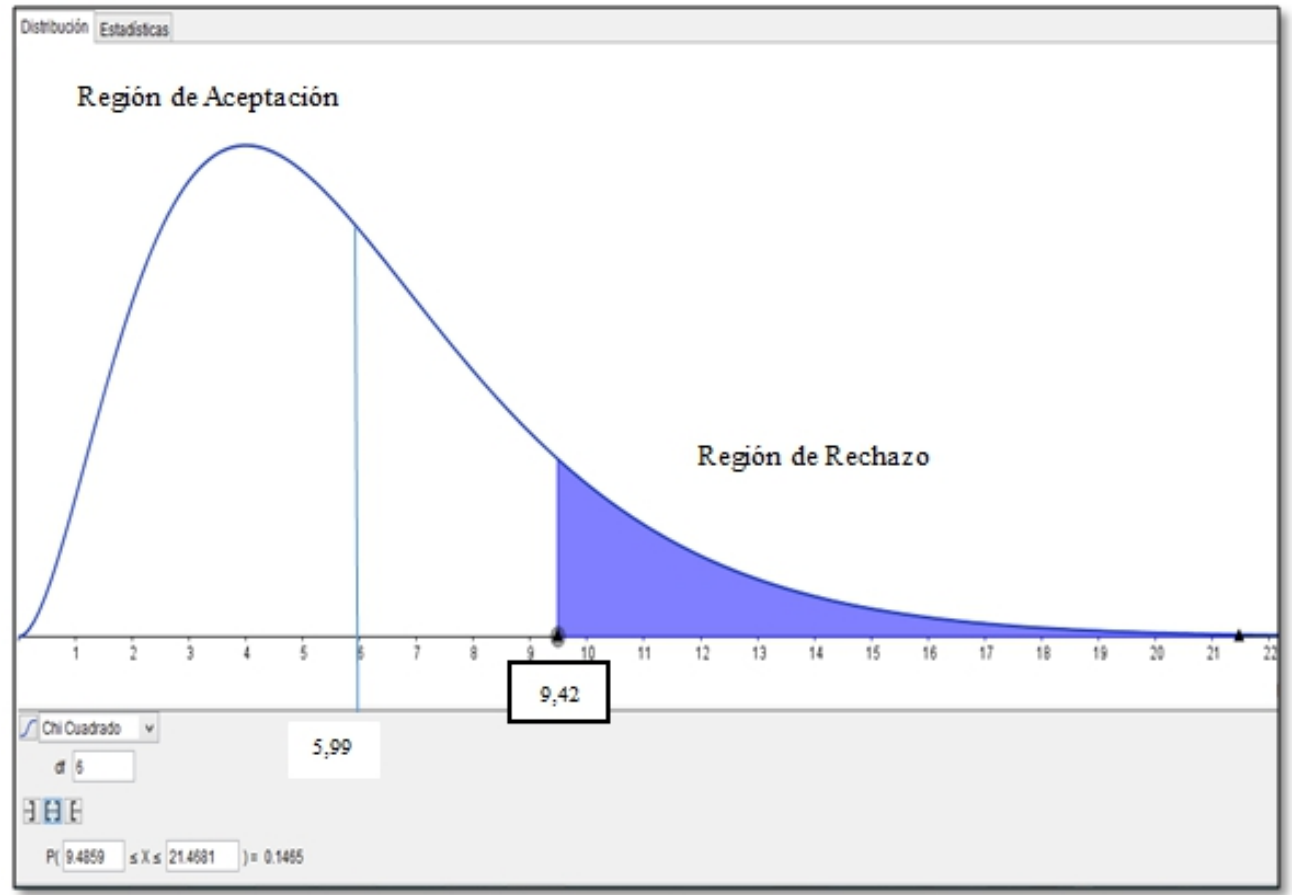

FiGura 1. Campana De Gauss 


\section{Recolección de Datos y Cálculo de lo Estadístico}

Para el cálculo se tomó en cuenta todas las preguntas, con los siguientes datos:

Encuesta aplicada a Entrenadores: Circuito 06D01C02 del distrito Chambo-Riobamba, Provincia de Chimborazo

Tabla 25.

\begin{tabular}{|c|c|c|c|c|}
\hline \multirow{2}{*}{ Preguntas } & \multicolumn{2}{|c|}{$\begin{array}{c}\text { Entrenadores Circuito 06D01C02 del distrito Chambo-Riobamba, } \\
\text { Provincia de Chimborazo. }\end{array}$} & \multirow{2}{*}{ Total } \\
\cline { 2 - 4 } & Siempre & Casi siempre & Nunca & \\
\hline 1 & 4 & 0 & 2 & 6 \\
\hline 2 & 5 & 0 & 1 & 6 \\
\hline 3 & 5 & 0 & 2 & 6 \\
\hline 4 & 4 & 0 & 1 & 6 \\
\hline 5 & 5 & 0 & 1 & 6 \\
\hline 6 & 5 & 0 & 2 & 6 \\
\hline 7 & 4 & 0 & 2 & 6 \\
\hline 8 & 4 & 0 & 3 & 6 \\
\hline 9 & 3 & 0 & 5 & 6 \\
\hline 10 & 1 & 0 & 20 & 60 \\
\hline Total & 40 & 0 & & 6 \\
\hline
\end{tabular}

Nota: Análisis a partir de los resultados de las encuestas

Encuesta aplicada a los estudiantes del Circuito 06D01C02 del distrito Chambo-Riobamba, Provincia de Chimborazo

Tabla 26.

\begin{tabular}{|c|c|c|c|c|}
\hline \multirow{2}{*}{ Preguntas } & \multicolumn{3}{|c|}{ Estudiantes Circuito 06D01C02 del distrito Chambo-Riobamba, } & Total \\
& \multicolumn{3}{|c|}{ Provincia de Chimborazo. } & \\
\cline { 2 - 5 } & Siempre & Casi siempre & Nunca & \\
\hline 1 & 125 & 1 & 14 & 140 \\
\hline 2 & 138 & 1 & 2 & 140 \\
\hline 3 & 137 & 1 & 1 & 140 \\
\hline 4 & 138 & 1 & 6 & 140 \\
\hline 5 & 133 & 1 & 5 & 140 \\
\hline 6 & 133 & 2 & 4 & 140 \\
\hline 7 & 134 & 2 & 6 & 140 \\
\hline 8 & 133 & 1 & 3 & 140 \\
\hline 9 & 134 & 3 & 18 & 140 \\
\hline 10 & 115 & 7 & 60 & 1400 \\
\hline Total & 1320 & 20 & 140 & \\
\hline
\end{tabular}

Nota: Análisis a partir de los resultados de las encuestas 


\section{FRECUENCIAS OBSERVADAS}

Tabla 27.

\begin{tabular}{|c|c|c|c|c|}
\hline \multirow{2}{*}{ PREGUNTAS } & \multicolumn{3}{|c|}{ CATEGORÍAS } & \multirow{2}{*}{ TOTAL } \\
\cline { 2 - 4 } & Siempre & Casi siempre & Nunca & \\
\hline Entrenadores & 4 & 0 & 2 & 6 \\
\hline Estudiantes deportistas & 132 & 2 & 6 & 140 \\
\hline TOTAL & 136 & 2 & 8 & 146 \\
\hline
\end{tabular}

Nota: Análisis a partir de los resultados de las encuestas

\section{FRECUENCIAS ESPERADAS}

\begin{tabular}{|c|c|c|c|c|}
\hline \multirow{2}{*}{ PREGUNTAS } & \multicolumn{3}{|c|}{ CATEGORÍAS } & \multirow{2}{*}{ TOTAL } \\
\cline { 2 - 4 } & Siempre & Casi siempre & Nunca & \\
\hline Entrenadores & 5,59 & 0,08 & 0,33 & 6 \\
\hline Estudiantes deportistas & 130,41 & 1,92 & 7,67 & 140 \\
\hline TOTAL & 136 & 2 & 8 & 146 \\
\hline
\end{tabular}

Nota: Análisis a partir de los resultados de las encuestas

Tabla 29. CÁLCULO DEL JI-CUADRADO

\begin{tabular}{|c|c|c|c|c|}
\hline $\mathrm{O}$ & $\mathrm{E}$ & $\mathrm{O}-\mathrm{E}$ & $(\mathrm{O}-\mathrm{E}) 2$ & $(\mathrm{O}-\mathrm{E}) 2 / \mathrm{E}$ \\
\hline 4 & 5,59 & $-1,59$ & 2,5251 & 0,45 \\
\hline 0 & 0,08 & $-0,08$ & 0,0068 & 0,08 \\
\hline 2 & 0,33 & 1,67 & 2,7930 & 8,50 \\
\hline 132 & 130,41 & 1,59 & 2,5251 & 0,02 \\
\hline 2 & 1,92 & 0,08 & 0,0068 & 0,00 \\
\hline 6 & 7,67 & $-1,67$ & 2,7930 & 0,36 \\
\hline 146 & 146 & & & 9,42 \\
\hline
\end{tabular}

Nota: Análisis de la investigación

\section{Decisión Final}

Para 2 grados de libertad a un nivel de $\mathbf{0 , 0 5}$ se obtiene en la tabla $\mathbf{5 , 9 9}$ y como el valor del ji-cuadrado calculado es $\mathbf{9 , 4 2}$ se encuentra fuera de la región de aceptación, entonces se rechaza la hipótesis nula por lo que se acepta la hipótesis alternativa que dice: "Los fundamentos básicos técnicos del baloncesto inciden en el rendimiento deportivo de los estudiantes categoría intermedia del circuito 06D01C02 distrito Chambo - Riobamba, provincia de Chimborazo. Por tanto se comprueba la Hipótesis como verdadera

\section{Discusión}

El entrenamiento básico según MATVEIEV, 1980 debe estar orientado a crear una base variada orientada hacia los diferentes deportes sean estos de conjunto o con pelota, combate, de arte y apreciación y de 
tiempo y marca orientados al desarrollo de la coordinación psicomotriz y la resistencia general sin volumen e intensidad

Para Fromenta (2003), dentro de la parte metodológica que pertenece a la fase formativa del jugador, se deben planificar y realizar actividades simples, las mismas que contribuyan sobre el aprendizaje significativo de algunos elementos técnicos, que debe dominar el basquetbolista, el objetivo primordial en esta etapa va encaminado a favorecer el desarrollo motivacional por la práctica del deporte, el conocimiento práctico y teórico de los fundamentos técnicos y tácticos, indispensables dentro del baloncesto, como son: el dribling los diferentes tipos de desplazamientos ,y pases del balón, el entrenador debe prestar vital atención a las diferencias individuales de cada uno de sus jugadores, como parte fundamental dentro del proceso de enseñanza-aprendizaje, y a la utilización del balón como recurso metodológico para el aprendizaje de los gestos técnicos (Echavarría, 2002).

Taureaux (2013) menciona que la consideración didáctica metodológica fundamental sobre la cual se basa la metodología, se relaciona con el hecho de que en la actualidad existe una utilización desmedida de un modelo de enseñanza puramente técnico - memorístico por los entrenadores de baloncesto en la categoría infantil; esta problemática como resultante del diagnóstico realizado nos sitúa en posición de dudar acerca de la efectividad que posee la metodología actual de la enseñanza de los fundamentos técnico tácticos de la categoría infantil 11-12 años del baloncesto.

Como sugerencia el autor Moya (1998) menciona que dentro de cada actividad psicomotriz o ejercicio planificado, no debería realizarse solo por adaptación momentánea, lo que permitirá que sobre el desarrollo de un ejercicio, el entrenador tenga la posibilidad de planificar actividades con objetivos similares, de esta manera el jugador podrá tener a su haber un sinnúmero de situaciones propias de la competencia y así poder extrapolar los movimientos obtenidos durante su formación deportiva.

De acuerdo a Alarcon (2011), los elementos didácticos que deben predominar en la formación de las Unidades Didácticas deberán estar en concordancia con el concepto constructiva del aprendizaje, para lo cual el entrenador deberá tomar en cuenta el instante de desarrollo motor del deportista y cada una de las características personales, ya que no todos se encuentran al mismo nivel para aprender ciertos contenidos en un período de edad concreta. Este nuevo aprendizaje debe ir en secuencia de una evaluación inicial, misma que pondrá de manifiesto el grado de desarrollo motor y los conocimientos adquiridos previamente que domina el deportistaalumno, y que guiaran al entrenador-profesor a realizar los ajustes necesarios en las secuencias que se presentan las actividades de aprendizaje. 


\section{Conclusion}

- $\quad$ Se determinó que los fundamentos básicos técnicos del baloncesto de la categoría intermedia son: el dribling, el tiro y los pases mismos que se ponen de manifiesto durante mayor parte del juego y se deberían trabajar con énfasis en el desarrollo y perfeccionamiento de los mismos.

- Después del análisis de los resultados podemos analizar que el nivel de rendimiento deportivo de los estudiantes deportistas de la categoría intermedia del circuito 06D01C02 distrito Chambo-Riobamba, de la provincia de Chimborazo es considerablemente bajo debido a las deficiencias técnicas observadas y al poco trabajo que se le brinda para el desarrollo de los mismos.

\section{References:}

1. Alarcon, F. (2011). La metodologia de la enseñanza en los deportes de conjunto. Revista de Investigacion en Educacion, 93-110.

2. Ecuador, A. N. (2010). Ley del Deporte, Educacion Fiisica y Recreación. Quito: Tribunal Costitucional de la Republica del Ecuador.

3. Frometa, E. (2003). La enseñanza de la Tecnica de las Carreras en la Etapa de Iniciación . efdeportes, 1.

4. MATVEIEV. (1980). Fundamentos del Entrenamiento Deportivo. Madrid: Raguda.

5. Moya, F. E. (1998). El concepto de la Tactica Individual en los deportes colectivos . Apunts , 16-22.

6. Echavarría, J.(2002). El Proceso de Enseñanza - Aprendizaje. Efedeportes, 1.

7. Taureaux, R. (2013). Metolodogia para la enseñaza de los Fundamentos Tecnico Tacticos del Baloncesto categoria Infantil 1112 años en el Municipio de Santiago de Cuba. efdeportes, 1. 
Anexos

ANEXO II
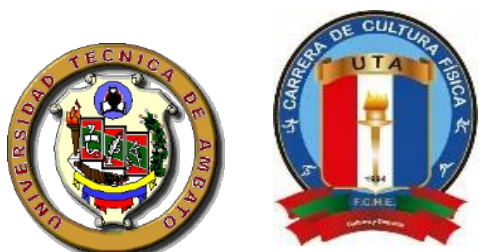

UNIVERSIDAD TÉCNICA DE AMBATO

Facultad Ciencias Humanas y de la Educación

Maestría en Cultura Física y Entrenamiento Deportivo

Encuesta 1. Dirigida a Entrenadores del "Circuito 06D01C02 del Distrito Chambo - Riobamba de la Provincia De Chimborazo"

OBJETIVO: Valorar el conocimiento de los entrenadores de baloncesto del "Circuito 06D01C02 del distrito Chambo-Riobamba de la provincia de Chimborazo" sobre la enseñanza de los fundamentos básicos técnicos del baloncesto la Práctica del baloncesto y el rendimiento deportivo.

1.- ¿La exactitud de los fundamentos técnicos de baloncesto permite el mejor desenvolvimiento en el juego?

Siempre

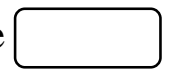
Casi siempre

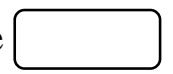

Nunca

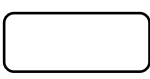

2.- ¿Usted como entrenador considera que la rapidez de movimiento permite ejecutar las acciones con eficiencia y eficacia?

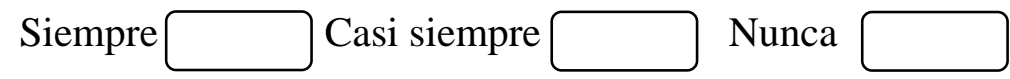

3.- ¿Cree usted como entrenador que el dribling, el tiro, y los pases son indispensables en el juego?

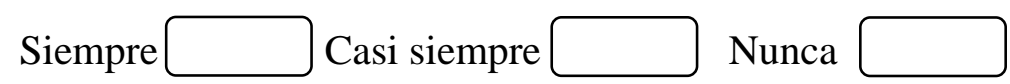

4.- ¿El desarrollo de los fundamentos técnicos del baloncesto permite el buen desempeño en el campo de juego?

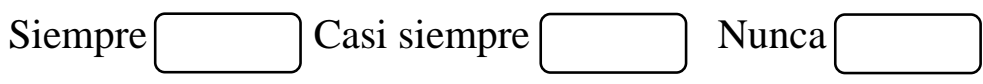

5.- ¿Cree usted que el deportista debe conocer acerca del arbitraje?

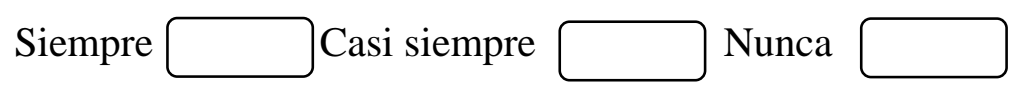

6.- ¿Cómo indicador potencial la técnica influye en el rendimiento deportivo?

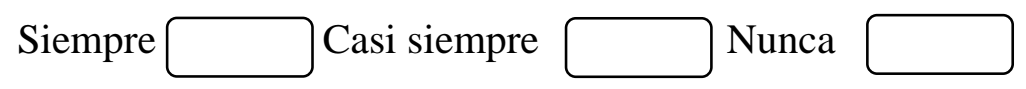


7.- ¿Cree usted que la fuerza, velocidad y resistencia son capacidades física predominantes del rendimiento deportivo?

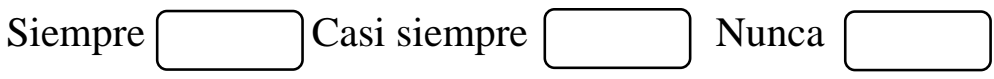

8.- ¿Considera importante trabajar la flexibilidad para mejorar el rendimiento deportivo en los estudiantes?

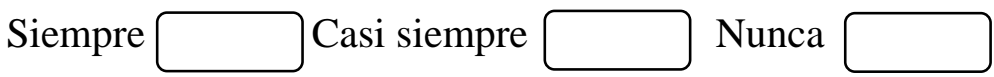

9.- ¿La preparación psicológica permite una buena estabilización en el rendimiento deportivo?

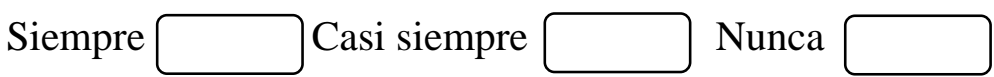

10.- ¿Cree usted que la aplicación de un plan de entrenamiento mejora su rendimiento deportivo?

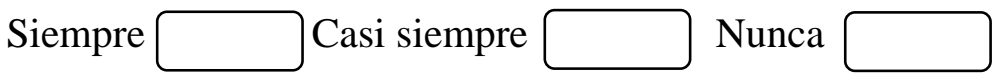

Encuesta 2

UNIVERSIDAD TÉCNICA DE AMBATO

FACULTAD CIENCIAS HUMANAS Y DE LA EDUCACIÓN

MAESTRIA EN CULTURA FISICA Y ENTRENAMIENTO

DEPORTIVO

Encuesta dirigida a estudiantes deportistas del "circuito 06d01c02 del distrito Chambo-Riobamba de la provincia de Chimborazo"

OBJETIVO: Conocer el criterio de los deportistas de baloncesto del "Circuito 06D01C02 del distrito Chambo-Riobamba de la provincia de Chimborazo" sobre el aprendizaje de los fundamentos básicos técnicos del baloncesto y el rendimiento deportivo.

1.- ¿La exactitud de los fundamentos técnicos de baloncesto permite el mejor desenvolvimiento en el juego?

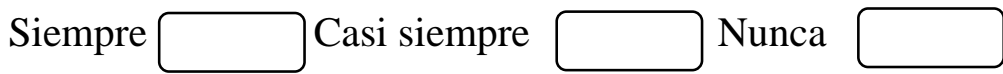

2.- ¿Usted como deportista considera que la rapidez de movimiento permite ejecutar las acciones con eficiencia $y$ eficacia? 
Siempre
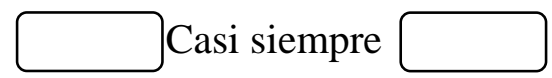

Nunca

3.- ¿Cree usted como deportista que el dribling, el tiro, y los pases son indispensables en el juego?

Siempre

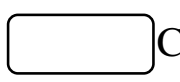
Casi siempre

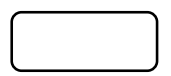
Nunca

4.- ¿EI desarrollo de los fundamentos técnicos del baloncesto permite el buen desempeño en el campo de juego?

Siempre

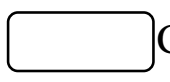

Casi siempre

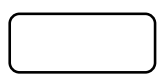

Nunca

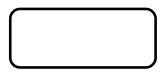

5.- ¿Cree usted como deportista que debe conocer acerca del arbitraje?

Siempre

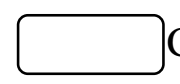
Casi siempre

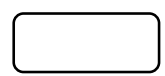

Nunca

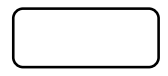

6.- ¿Cómo indicador potencial la técnica influye en el rendimiento deportivo?

Siempre

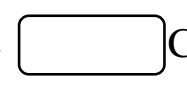
Casi siempre

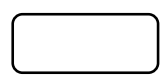
Nunca

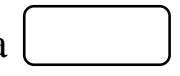

7.- ¿Cree usted que la fuerza, velocidad y resistencia son capacidades física predominantes del rendimiento deportivo?

Siempre

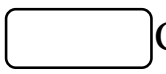
Casi siempre

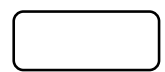

Nunca

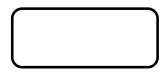

8.- ¿Considera importante trabajar la flexibilidad para mejorar el rendimiento deportivo en los estudiantes?

Siempre
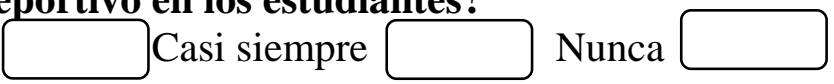

9.- ¿La preparación psicológica permite una buena estabilización en el rendimiento deportivo?

Siempre
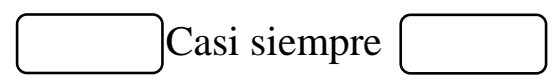

Nunca

10.- ¿Cree usted que la aplicación de un plan de entrenamiento mejora su rendimiento deportivo?

Siempre
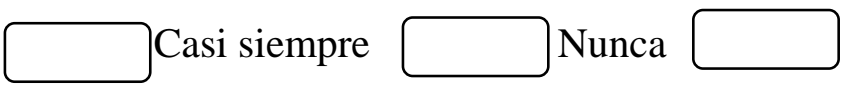